\title{
Summary of: Knowledge of removable partial denture wearers on denture hygiene
}

\section{FULL PAPER DETAILS}

${ }^{1}$ Cardiff University, School of Dentistry, Heath Park Cardiff, CF4 4XY

${ }^{*}$ Correspondence to: Mr Paul Milward Email:milward@cardiff.ac.uk

Refereed Paper

Accepted 29 July 2013

DOI: 10.1038/sj.bdj.2013.1095

British Dental Journal 2013; 215: E20

\author{
P. Milward, ${ }^{* 1}$ D. Katechia ${ }^{1}$ and M. Z. Morgan ${ }^{1}$
}

Background Regular good denture hygiene by individuals with removable partial dentures (RPDs) is an important component of oral health and in the prevention of further dental problems. These individuals should be provided with advice on the importance of denture care and be aware of this information. Aim To establish deficiencies in patient knowledge surrounding denture hygiene by RPD wearers. Methodology The study was undertaken as an audit. Data was collected from April 2012 to October 2012 via a questionnaire completed by 196 RPD wearers attending as patients at the University Dental Hospital Wales and the dental units at St David's Hospital and Cynon Valley Hospital. The audit criterion was patients with RPDs should have knowledge of denture hygiene, with the standard set at 100\%. Results While 91.8\% of participants stated they were provided with instructions on denture hygiene when provided with their current prosthesis, $60.2 \%$ were shown to have less than an appropriate level of denture cleanliness, with $9.2 \%$ reporting that they slept wearing their prosthesis. Conclusion The audit criterion and standard set were not achieved. A lack of knowledge surrounding denture hygiene was demonstrated among participants. As a part of the audit process the health education of RPD wearers' hygiene needs to be improved and awareness levels of the whole dental team needs to be raised. All partial dentures should receive information and regular reinforcement of key dental hygiene messages.

\section{EDITOR'S SUMMARY}

Prosthetics, as it was, and prosthodontics as it is, largely remains the Cinderella of dentistry despite its name change. True, there has been a shift in the extent of its practice in the UK at least away from full-full cases to those of partial dentures and the increased complexity of dentures supported by implants but the fact that it is often seen as the poor relation is difficult to dispel.

It is also noticeable that we have few papers submitted and fewer still published in the $B D J$ which makes this one the more interesting. There is no doubt that the shift away from full dentures towards partials has been hastened by the improving oral health in the UK in recent years meaning, as is so frequently quoted, that more of us are keeping more teeth for longer. However, this paper focuses on those patients who have kept only some of their teeth for longer and are in need of prosthetic replacement of others.
As with peri-implantitis, one is tempted to query whether the clue to the question is in the initial problem. With the exception of trauma and some other causes of missing or absent teeth, the majority of tooth loss is due to either caries or periodontal disease and whether this has been occasioned by poor diet or poor oral hygiene the fact remains that the past care has been less than ideal.

Perhaps not surprising then that this paper uncovers a majority of partial denture wearers demonstrating less than optimum oral health and denture cleanliness. Further, the pathology almost tells its own story, as those who had 'additions' to their partial dentures were far worse at keeping them clean than those who did not. Presumably an ongoing self-fulfilling prophesy.

Clearly with the likelihood of greater partial denture provision in the future we have to show much greater awareness of the oral hygiene of patients so affected. It is an area in which the den- tal team can also play a greater part, in supporting and reinforcing the message from the main clinician in terms of the importance of continuing plaque control, denture care and pride in maintenance of good oral health.

The full paper can be accessed from the $B D J$ website (www.bdj.co.uk), under 'Research' in the table of contents for Volume 215 issue 10.

Stephen Hancocks Editor-in-Chief DOI: 10.1038/sj.bdj.2013.1118 


\section{TO ACCESS THE BDJ WEBSITE TO READ THE FULL PAPER:}

- BDA Members should go to www.bda.org.

- Click the 'login' button on the right-hand side and enter your BDA login details.

- Once you have logged in click the 'BDJ' tab to transfer to the BDJ website with full access.

IF YOUR LOGIN DETAILS DO NOT WORK:

- Get a password reminder: go to www.bda.org, click the login button on the right-hand side and then click the forgotten password link.

- Use a recommended browser: we recommend Microsoft Internet Explorer or Mozilla Firefox.

- Ensure that the security settings on your browser are set to recommended levels.

IF YOU HAVE NOT YET SIGNED UP TO USE THE BDA WEBSITE:

- Go to www.bda.org/getstarted for information on how to start using the BDA website.
IN BRIEF

- Reports that only $60 \%$ of partial denture wearers demonstrated appropriate levels of denture cleanliness.

- Informs that out of $44 \%$ of the participants who had further additions to their partial dentures only $10 \%$ had good levels of denture cleanliness.

- Proposes the need for dentists to increase the awareness of the importance of denture hygiene for partial denture wearers and their future oral health.

\section{COMMENTARY}

This article, an audit report, highlights a very important aspect of removable partial denture (RPD) provision, that of denture hygiene and patient knowledge regarding aftercare and revisit frequency.

The assessment of the level of cleanliness of RPDs worn by the patients in the audit was assessed against the criteria of: the number of times the RPD was cleaned daily, the type of material the RPD was constructed from, the frequency of attendance to a clinician for examination, the form in which instruction/advice on denture hygiene was given and whether participants had further additions to their dentures.

Some of the more interesting findings were:

- Dentures with additions showed poorer cleanliness than those without - Verbal advice by the clinician was the most common method of providing advice on denture aftercare. From the findings of this study verbal information should always be backed up with written advice - Water was the most popular cleaning medium

- Cobalt/chromium RPDs showed higher levels of cleanliness than acrylic resin RPDs.

The audit highlighted the lack of knowledge and information among patients regarding all aspects of RPD hygiene and the need to regularly attend for check ups.

Recommendations are given regarding improving the information/advice given by the clinician, when providing RPDs, to better educate the patient regarding oral hygiene methods available, the need to maintain good oral hygiene and the importance of regular attendance to the clinician to ensure no further oral damage develops.

\section{Dr Tony Johnson}

Senior Lecturer

Academic Unit of Restorative Dentistry

School of Clinical Dentistry

University of Sheffield

\section{AUTHOR QUESTIONS AND ANSWERS}

1. Why did you undertake this research? The research was undertaken to investigate the knowledge of current partial denture wearers about denture hygiene and maintenance in order to assess whether information is being given on this topic by dental professionals.

The aim was to identify if every partial denture wearer was being given appropriate instructions, information and advice in order to allow them to clean and care for their partial denture appropriately. The research also investigated the wearer's perception of the standard of hygiene of their dentures.

2. What would you like to do next in this area to follow on from this work?

This audit has provided information on differences in the denture cleaning habits and care regimes of removable partial denture wearers. Deficiencies in knowledge on this topic have been recognised and possible changes identified, which we would like to implement to improve patient and dental professionals' awareness of the importance of good partial denture hygiene. Future work will be to repeat the research on wearers provided with their dentures by GDPs. This could lead to a discussion amongst the dental profession on how to improve the knowledge and awareness of RPD wearers on denture hygiene in the future. To remedy this an improvement in the provision of information to removable partial denture wearers is necessary. In Cardiff and Vale NHS Trust and Cardiff University we propose to develop appropriate protocols for inclusion in undergraduate and postgraduate training courses. 